\title{
Pre-syncope and ataxia after spinning whiplash
}

\begin{abstract}
This is a 46year old female presenting to her Primary Care Physician on Day \#2 post-motor vehicle accident. She was the driver of a large suburban truck that was suddenly T-boned into her left drivers' rear wheel, whirling 360 degrees to face oncoming traffic. She and her daughter went home. But over time, the patient slept more and more. She experienced ataxia and pre-syncope. Dozens of presentations to the Emergency Department (ED) ensued. Eventually, she could not stand up without vertigo, ataxia, pre-syncope, and vomiting. After 2 months, the patient still had severe nausea and vomiting 12times/day, was weak, ataxic, confused, and had short-term memory loss, and developed tinnitus and an expressive aphasia. She still had presyncope on standing. She was diagnosed with a traumatic brain injury (TBI).
\end{abstract}

After 3 months, the patient underwent an MRI of head and neck without contrast, and it was negative. Ten ED visits later, the doctor ordered a spiral CAT scan that detected an extra cranial $3 \mathrm{~cm}$ left vertebral artery dissection (VAD). Angiogram confirmed the VAD at $\mathrm{C} 4-5$, with a $2 \mathrm{~cm}$ aneurysm at the base. Medical management failed to correct pre-syncope, so the patient kept "doctor shopping" until led to Electrophysiology Cardiologist, Dr. David Cannom, MD. She underwent the Tilt Table Test (TTT), passing out for 22seconds. The diagnosis of Dysautonomia was made. This was aggravated by polydypsea and polyuria, so she was diagnosed with diabetes insipidus (DI) and placed on a PICC line for almost four years.

After 7years and a nearly full recovery with a return to work as an anesthesiologist in sight, a neurologist allowed her fall to the hard wood floor during his "close your eyes and touch your nose" exam while standing; the patient sustained another TBI with DI and remains disabled.

Keywords: pre-syncope, vomiting, ataxia, traumatic brain injury, diabetes insipidus, vertebral artery dissection, dysautonomia, oculo-vestibular dysfunction, gastroparesis, syncope
Volume 4 Issue I - 2016

Margaret Aranda

Aranda MD Enterprises, USA

Correspondence: Margaret Aranda, Aranda MD Enterprises, 26500-102 Agoura Road, Suite 656 Calabasas, California, USA, Tel 818584933।,Email drmargaretaranda@yahoo.com

Received: December 25, 2015 | Published: January 22, 2016
Abbreviations: DI, diabetes insipidus; ED, emergency department; EPS, electrophysiology; ICU, intensive care unit; IV, intravenous; PICC, percutaneous intravenous central catheter; POTS, postural orthostatic tachycardia syndrome; TTT, tilt table test; TBI, traumatic brain injury; VAD, vertebral artery dissection

\section{Introduction}

This case highlights the issues of "physician patients," "selfdiagnosis, "malingering," dysautonomia, TBI with DI, and VAD. The question of whether some illnesses are "rare" vs "undiagnosed" is a key focus, as well as the proposed need for increased physician education on "invisible illnesses," so that patients are not distraught and made to feel mentally ill. The sequelae of depression, anxiety, and loss of patient dignity contribute greatly to decreased quality of life, and increased monetary strain on the health care system, which in some cases, is being used out of desperation, as a "revolving door." Failure to diagnose could contribute to physician frustration and loss of job enthusiasm.

\section{Case presentation}

While still a resident in Anesthesiology at the University Of Southern California School Of Medicine, the patient initiated the grant-writing and research of patients with intractable closed head injury in the Intensive Care Unit (ICU). It was published as a Rapid Communication in Neurosurgery. ${ }^{1}$ She continued academic medicine pursuits in the subjects including the Surgical ICU, ${ }^{2-5}$ pulmonary hypertension, ${ }^{6}$ nitric oxide, ${ }^{7,8}$ ventilation/perfusion mismatch,,${ }^{9,10}$ and Hyperpolarized ${ }^{3} \mathrm{He}-\mathrm{MRI}$ of the porcine lung. ${ }^{11-13}$ She also presented multiple Poster Presentations and was a leader in her field, Hyperpolarized ${ }^{3} \mathrm{He}-\mathrm{MRI}$ of the porcine lung, having internationally lectured on V/Q mismatch and ${ }^{3} \mathrm{He}-\mathrm{MRI}$ of the lungs. ${ }^{14,15}$ She collaborated with other anesthesiologists, radiologists, internal medicine physicians, and physicists on projects ${ }^{11-13}$ and helped write $\$ 3$ million in successful NIH Research Grants at the University of Pennsylvania.

Being keenly astute as to her symptoms and medical history, she was unprepared to undergo the path to her multiple "rare" diagnoses, yet was known for her "persevering" attitude. Prior to the car accident, her last job position was as anesthesiologist and Director of the SICU at a Veterans Administration in Southern California. Her highest position was that as Interim Chief of Anesthesiology at the Philadelphia Veteran's Administration in Pennsylvania, USA. She had no medical problems except seasonal allergic asthma, anaphylaxis to sun exposure, and anxiety due to job-related stress and a pregnancy that was carried to term, with elective Caesarian Section and no complications except for mastitis, for which she was treated with antibiotics.

This is to be clear that she was not only an intelligent physician, but an academic one who was used to defending her research by symposium podium or poster presentation, or lecture hall podium in Grand Rounds. She also had expertise in making quick and authoritative decisions both as Chief of Anesthesiology and in the 
Operating Rooms, as an anesthesiologist, and as an intensivist trained in the ICUs at Stanford. She had a noted reputation for being extremely ethical, compassionate to all of her patients, and for never "losing her cool."

\section{Discussion}

\section{TBI with DI}

The CDC estimates 1.7 million TBIs per year, with about $30 \%$ leading to death. This combination is associated and learned by every medical student, with there being no need for actual head impact for TBI to occur, as in whiplash. The patient presented with poor cognition, inability to drive without getting lost, forgetfulness, and an expressive aphasia. Physical exam findings included nystagmus and loss of proprioception when standing alone. This was also associated with nausea/vomiting, diagnosed as mal de Debarquement Syndrome. Both CT scan and MRIs were normal for brain anatomy.

\section{DI}

The incidence of severe DI in acute TBI is about $3 \%$. Secondary hypoadrenalism and hypothyroidism can occur in a secondary or later stage of TBI, with an incidence of about $10 \%$. There is a need to evaluate the pituitary: hypothalamus axis, the thyroid axis, and ADH secretion.

Each time, the DI was diagnosed by the patient. Polyuria and polydipsia were the primary presentations. With central or psychogenic DI, the posterior pituitary gland is traumatized, leading to a defect in the hypothalamic: pituitary gland axis, which regulates $\mathrm{ADH}$ and total body water. No ADH is produced. The diagnosis can be made by the water deprivation test, if the patient is not imminently close to renal failure from dehydration. This involves ${ }^{1}$ maintaining npo status, ${ }^{2}$ insertion of a Foley catheter, and ${ }^{3}$ water deprivation. In the first DI episode, the patient urinated 4liters urine in 2hours. On the second episode, she was dehydrated by lab results: $\mathrm{BUN} / \mathrm{Cr}=104 / 81$; $\mathrm{GFR}=35 \%$; platelet count of $45 \mathrm{~K}$ on generic clopidogrel $75 \mathrm{mg}$ (for the $\mathrm{VAD}$ ) and simply treated with $\mathrm{ADH}$ and resuscitation prior to renal insult. Resuscitation included NS fluid bolus plus $60 \mathrm{ml} / \mathrm{hr}$; IV steroids, meclizine, and head down flat. Legs were elevated for a mean arterial pressure less than $60 \mathrm{~mm} \mathrm{Hg}$.

Treatment of DI was with anti-diuretic hormone (ADH) po, (AKA vasopressin), AKA DDAVP via nasal spray. The underlying dysautonomia caused titration problems so that sometimes the seesaw tipped her sodium to $158 \mathrm{mcg} / \mathrm{ml}$ and she was dehydrated; other times, her sodium was $127 \mathrm{mcg} / \mathrm{ml}$ and she felt like a water balloon about to undergo uncal herniation and eyeball expulsion. Both nasal and po DDAVP were problematic as treatment therapies, due to the underlying dysautonomia as a hypovolemic state. The patient relied on daily weights and the color of her urine (clear water vs. dark yellow) to dictate the next dose of DDAVP, especially overnight. Her chief complaint was dry mouth, which required more frequent dental visits to avoid periodontitis due to lack of sterilizing saliva. This put she at risk of cerebral stroke, especially since laminar flow in the vertebral artery dissection area could not be guaranteed, tending towards coagulation. Each time, the DI resolved after about 6months.

\section{Dysautonomia}

The incidence of orthostatic hypotension is age-dependent, with patients under 50yo having a 5\% incidence, and patients over 70 yo having a $30 \%$ incidence. The patient experiences significant quality of life decreases, with increased mortality and incidence in heart attack, stroke, heart failure, and atrial fibrillation. ${ }^{16}$

In The ED, patient presentations are wrought with disgust and dread. Doctor after doctor did ortho statics on the patient, with her standing numbers going as low as a blood pressure of $75 / 44 \mathrm{mmHg}$, with a heart rate of $110 \mathrm{bpm}$. When she was unable to stand up for fear of syncope, one doctor said he was "going to look it up in my medical school books" and came in later to say, "You're supposed to wait 5 minutes before taking the standing blood pressure." The patient said, "Do you want to scrape me off the floor? I won't last that long!" There was a large dichotomy between physician assessment/ impression and patient complaints/experience. ${ }^{17-19}$ Only because one doctor knew another doctor who "worked with patients who faint," did the patient finally get a referral to an EPS cardiologist. The most common presentation of orthostatic intolerance is in a female (80\%) with Postural Orthostatic Tachycardia Syndrome (POTS), which is defined as sustained increase in heart rate by $30 \mathrm{bpm}$ at 10 minutes after standing, with an increase in noradrenalin serum concentration. ${ }^{20}$

The patient was checked in to the Outpatient Cardiology Suite for the state-of-the-art diagnostic tool, the Tilt Table Test. She complained of "going gray" when the table was lifted from the supine position to a $90^{\circ}$ standing angle. She had complete syncope with loss of consciousness for 22seconds, without seizure activity or loss of bowel/bladder function. The definitive diagnosis of Dysautonomia was made. The pathophysiology of POTS and other dysautonomias is an underlying cerebral hypo perfusion upon standing, with lack of vasoconstrictor alpha-1 agonist activity in the legs.

Since then, she has been on the alpha-agonist Midodrine to increase her blood pressure, she wears Jobst ${ }^{\circledR}$ Stocking thigh-highs, and an abdominal binder. All of these increase cerebral blood flow and allow perfusion to the brainstem primary autonomic nervous system centers, allowing her to sit and stand for small periods of time. She has spent most of the last 9years in bed.

Only in the cardiac hospital, Good Samaritan, where Dr. David Cannom practices with many patients with Dysautonomia, was she 'fast-tracked' into the ER, into admissions, into getting an iv, consults, and getting her brain re-perfused with bolus NS, iv steroids, meclizine, ondansatron, and dilaudid as "her" recipe back to health. After 4years, the PICC line was removed. But the dysautonomia kept her in a constant hypovolemic state by nature, and she was taking $10 \mathrm{mg}$ Midodrine every morning, and another $10 \mathrm{mg}$ in the afternoons. For nausea/vomiting, she was on IV Ondansetron $4 \mathrm{mg}$ bid for 3years, then on $4 \mathrm{mg}$ /day until now. Endocrine workup showed a diagnosis of hypothyroidism, which was treated with $1 / 2$ gr Armour Thyroid.

Even after the diagnosis of Dysautonomia was made, not one doctor had ever heard of "Dysautonomia," so the reactions were genuinely a social study to observe. Some doctors pretended to know what it was, whereas others just looked blankly at the patient. Only a few asked for an explanation. If an unsolicited explanation was offered to benefit patient care, all smiles turned to frowns and the patient was treated as a "malingerer." Her requests for 1liter of NS bolus were ignored, as were her requests for treatment of a migraine headache. She had to leave the ER by wheelchair without any treatment on several occasions for lack of any treatment at all. She successfully argued against paying the ED insurance premium for lack of treatment. All in all, though, it was humiliating. 


\section{Vertebral artery dissection with aneurysm}

Extra cranial vertebral artery aneurysms are rare and not well described. For a small subset of patients with underlying hereditary or connective tissue disorder, operative intervention may be a choice through exclusion and reconstruction. The author surmises that many VADs simply go undiagnosed due to patient death.

Once the spiral CT detected a left vertebral artery abnormality, the patient was sent by ambulance to a major medical center for an angiogram of the brain. Upon angiogram, she recalls the injection and the warmth of the dye going through the Circle of Willis and back down the contralateral side. The extracranial LVAD was further identified as being $3 \mathrm{~cm}$ long, with a $2 \mathrm{~cm}$ aneurysm at the base. Lots of discussion was held over treatment for VAD. Very little literature exists on this subject, and the multi-center studies still had a very small "n." The final treatment plan was medical management without surgery or stenting: clopidogrel $75 \mathrm{mg} /$ day and Lipitor $80 \mathrm{mg} /$ day was started as a daily regimen for life. Over time, the Lipitor had decreased to $20 \mathrm{mg}$ /day, and after the second TBI, the generic clopidogrel was discontinued for Trade-Name Plavix ${ }^{\circledR} 75 \mathrm{mg} /$ day for life.

The doctor was now the patient, and she was confused from Day \#1. The ensuing events were remarkable not only for the fact that multiple, rare diagnoses were made, but that she had to "Speak up" and "Stand up" for her rights as a patient, refusing to be abandoned in a nursing home. She refused to be treated as a "drug-seeker." She knew to ask for the Charge Nurse, and then she knew to ask for the Incident Report after the doctor allowed her to fall to the floor, sustaining not only the second TBI with DI, but also requiring Left Shoulder Rotator Cuff Repair for falling onto her left side and protecting her head from hitting the floor.

After 4weeks of Neurorehabilitation and learning how to walk and talk again in 2006, the patient slowly recovered. In 2013, she had been accepted into Stanford's Advanced Fellowship for Clinical Anesthesiology to "get my stethoscope back," but the $2^{\text {nd }}$ TBI put this permanently out of reach.

It behooves physicians to listen to their patients, to take them seriously, and to refer them to another specialist (instead of sending them home empty-handed) if they are unable to make a diagnosis. Sometimes, physicians really need to "wrack their brains" hard, to find some solution for the patient. Some patients suffer for weeks, months, years, or decades until finally getting an "invisible illness" diagnosed by the $30^{\text {th }}$ doctor! May this never be you, or one of your loved ones?

By increasing physician education on a handful of "invisible illnesses, ${ }^{20}$ many more diagnoses can be made in a timely manner, more referrals to the proper specialists can be made, and both patient and physician frustration should be replaced by the genuine joy and hope that may be otherwise lacking in this scenario. This testimony is an inspiration to both doctor and patient alike, for "Perseverance" trumps medical textbooks and medical Case Reports every time.

The patient is still disabled, has severe orthostatic hypotension, and some cognitive dysfunction is expected to remain. She has become a published author and avid Patient Advocate for "Invisible Illnesses," serving on the Advisory Board to the Invisible Disabilities Association, which awarded her the 2011 Perseverance Award. She is a Public Figure on Facebook, and much loved by millions of disabled people who felt that doctors were simply "out of touch" and not sympathetic to the plight of those whose diagnosis may be best determined by another specialist. So, doctor, just because you haven't heard of a disease, it doesn't mean that it doesn't exist. Stanford's Medicine-X program encourages patients with invisible or rare diseases to join internet groups, become "e-patients," then "e-scholars" who help other patients to self-diagnose a rare disease. So don't squeak and squawk if your patient knows more about Chiari 1 malformation than you do. In today's world, she has to know more in order to survive. Support her, as we are all working toward the same goal: health, longevity, and quality of life.

\section{Acknowledgements}

None.

\section{Conflict of interest}

Author declares that there is no conflict of interest.

\section{References}

1. Levy M, Margaret A, Zelman V, et al. Propylene glycol toxicity following continuous etomidate infusion for the control of refractory cerebral edema. Neurosurgery. 1995;37(2):363-371.

2. Margaret A, Ronald GP. Pharmacology and physiology of nitric oxide. Anesthesiology Clinics. 1998;16(1):235-257.

3. Hanson CW, Margaret A. Impact of intensivists and ICU teams on patient outcomes. Journal of Intensive Care Medicine. 1999;14(6):254-261.

4. Aranda M, Hanson CW. Anesthetics, sedatives and paralytics Understanding their use in the intensive care unit. Surg Clin North Am. 2000;80(3):933-947.

5. Aranda M, Bradford, Katherine K, et al. Continuous therapy with inhaled nitric oxide and intravenous vasodilators during experimental pulmonary hypertension. Anesthesia \& Analgesia. 1999;89(1):152-158.

6. Aranda M, Ronald GP. The biology of nitric oxide. Resp Care. 1999;44(2):156-168.

7. Aranda M, Ronald GP. Inhaled nitric oxide and pulmonary vasoreactivity Journal of Clinical Monitoring and Computing. 2000;16(5):393-401.

8. Roberts DA, Rizi RR, David AL, et al. Dynamic observation of pulmonary perfusion using continuous arterial spin-labeling in a pig model. J Magn Reson Imaging. 2001;14(2):175-180.

9. Rizi RR, Saha PK, Wang B, et al. Co-registration of acquired MR ventilation and perfusion images -validation in a porcine model. Magnetic Resonance in Medicine. 2003;49(1):13-18.

10. Roberts DA, Rizi RR, Lipson DA, et al. Detection and localization of pulmonary air leaks using laser-polarized Helium-3 MRI. Magnetic Resonance in Medicine. 2000;44(3):379-382.

11. Ferrante M, Asiaii Ali, Ishii M, et al. An Evaluation of Pulmonary Atelectasis and its Re-expansion: Hyperpolarized ${ }^{3} \mathrm{HE}$ MRI in the Yorkshire Pig. Acad Radiol. 2003;10(11):1283-1290.

12. Markstaller K, Eberle B, Kauczor HU, et al. Lung density in dynamic CT correlates with oxygenation during pressure-controlled ventilation of pigs with lavage-ARDS. Br J Anaesth. 2003;91(5):699-708.

13. Rizi RR, David AR, Peter KS, et al. Atelectasis: A useful evaluation by hyperpolarized ${ }^{3}$ Helium magnetic resonance imaging. USA: University of Pennsylvania; Proc Intl SOC Mag Reson Med. 2002

14. Wang B, Saha PK, Udupa JK, et al. 3D airway segmentation via hyperpolarized ${ }^{3} \mathrm{He}$ gas MRI using scale-based fuzzy connectedness. Computerized Medical Imaging and Graphics. 2004;28(1-2):77-86. 
15. Ricci F, De Caterina R, Fedorowski A. Orthostatic Hypotension: Epidemiology, Prognosis, and Treatment. $J$ Am Coll Cardiol. 2015;66(7):848-860.

16. Ferrante, Margaret A, David S. Cannom featured: "Dysautonomia: Electrophysiologists Aids Patient with Uncommon Malfunction of the Central Nervous System." Good Samaritan Hospital Quarterly Summary, Page 3. 2009.

17. Ferrante, Margaret Aranda, David S. Cannom featured Featured: Denise Dador Reporting: "Dysautonomia" shown on KABC Eyewitness News Los Angeles; 2009.
18. Ferrante, Margaret Aranda, Davis S. Featured: "Dysautoomia: Patient Attacked by Rare Condition." Denise Dador Reporting. Channel 7 KABC News TV clip. 2009.

19. Rek M, Kaczmarek K, Cygankiewica I, et al. Postural Orthostatic Tachycardia Syndrome (POTS) --Pathophysiology, Diagnostics, and Treatment. Przegl Lek. 2014;71(8):450-453.

20. Walsh K, Hoffmayer K, Hamdan MH. Syncope: Diagnosis and Management. Curr Probl Cardiol. 2015;40(2):51-86. 